Research Article

\title{
No Gender Difference in Foreign Medical Students' Hematology Clerkship
}

\author{
Qing Li, ${ }^{1}$ Jianhui Chen, ${ }^{2}$ Bo Wan, ${ }^{3}$ and Xiaofan $\mathrm{Li} \mathbb{1 D}^{2,4}$ \\ ${ }^{1}$ Fujian Medical University Provincial Clinical College, Fujian Provincial Hospital, Fuzhou, China \\ ${ }^{2}$ Fujian Medical University Union Hospital, Fuzhou, China \\ ${ }^{3}$ The First Affiliated Hospital of Fujian Medical University, Fuzhou, China \\ ${ }^{4}$ Fujian Institute of Hematology, Fujian Provincial Key Laboratory on Hematology, No. 29, Xinquan Road, Fuzhou, Fujian \\ 350000, China
}

Correspondence should be addressed to Xiaofan Li; morningshiplee@fjmu.edu.cn

Received 19 February 2017; Revised 21 September 2017; Accepted 30 January 2018; Published 13 March 2018

Academic Editor: Gwo-Jen Hwang

Copyright ( 2018 Qing Li et al. This is an open access article distributed under the Creative Commons Attribution License, which permits unrestricted use, distribution, and reproduction in any medium, provided the original work is properly cited.

Hematology is a difficult aspect of medicine for students to learn. Students need hematology clerkship training to develop their clinical skills. The influence of gender on different teaching methods and performance in foreign medical students is still unknown, especially in hematology clerkship. To address this issue, we tested performance differences between foreign male and female students in clinical practice. Twenty-two foreign medical students attended a two-week hematology clerkship in Fujian Medical University Union Hospital. The lecture-based learning (LBL) method and mixed team-based learning (MTBL) method were used in hematology clerkship. Eight foreign students were assigned to LBL group, and fourteen foreign medical students were assigned to MTBL group. Our previous work found that MTBL methods benefit both foreign medical students and Chinese postgraduates. In this study, practice scores and case-writing scores were analyzed between boys and girls. The results showed that the mean of scores in practice test scores and case writing scores was not significantly different between genders $(P>0.05)$, as well as subgroup analysis in LBL and MTBL groups $(P>0.05)$. This study indicated that practice test scores and case-writing scores in hematology clerkship were the same between boys and girls, which were not related to teaching methods. This study also indicated that both genders can benefit from the new MTBL teaching methods.

\section{Introduction}

Gender issues in education have been a hot topic in medical education [1-4]. Hematology is one of the highly specialized subjects in medicine [5], which is a branch of medicine studying the diagnosis, treatment, and prevention of blood diseases. It includes problems with the red blood cells, white blood cells, platelets, blood vessels, bone marrow, lymph nodes, spleen, and the proteins involved in bleeding and clotting (hemostasis and thrombosis). Some students perceive hematology difficult. Indeed, the routine work of hematology includes both clinical practice and lab work. Some laboratory works of hematology will also be discussed during internship to help the students become more familiar with this area. For the beginners who have just entered clinical clerkship, it is important to choose appropriate teaching methods, especially for their adaptation to clinical.

The patients with hematological diseases are often serious. It is usually quickly progressed. Chemotherapy, radiotherapy, and hematopoietic stem cell transplantation are often used in the treatment of hematological disease patients, which can also affect the whole body. The combination of hematological knowledge, English lecture teaching, and clinical practice patterns during ward rounds has greatly promoted the foreign students' study [6].

In recent years, molecular biology and genetics related techniques are developing rapidly. Physicians need to have not only professional skills but also strong laboratory skills. Clerkship is a bridge to connect medical theory and clinical practice, which is a crucial stage in medical college. It is during 
their clerkships that medical students' clinical and practical skills are developed [7]. Gender difference affects lots of areas of education. However, whether there are gender differences in students of hematology is still unknown.

The past decades have witnessed a great increase of the number of international students at colleges and universities in China due to its opening-up policy. One of the widely used teaching methods in hematology clerkship is the lecture-based learning (LBL) method [8]. Fujian Medical University has international students. English medical classes were given to those international students. Fujian Institute of Hematology recruits Chinese postgraduate students who are willing to help international students to overcome the aforementioned gap in communication. In our previous studies, we designed a mixed team-based (mixed foreign students with local graduates) learning (MTBL) method for hematology clerkship, which benefits both foreign medical students and Chinese postgraduates in order to overcome communication problems in Chinese clinics [9]. However, the performance difference between genders in hematology clerkship, especially in MTBL, is unknown.

To evaluate the performance difference between foreign male and female students in hematology clerkship, proper preparation before clerkship is important. According to the characteristics of the foreign students, teachers were trained by Stanford-SUMC Clinical Faculty Development Workshop or FMU foreign language training class (https://www.cfd. med.stu.edu.cn/). These clinical teaching courses can enhance versatility for a teacher to improve the analyzing ability of teaching outcomes. Seminars of educational categories (including self-brief mini lectures) were given, and the general process of this training class includes the following: climate of learning, communication goals, and understanding promotion, feedback, and evaluation.

In this current study, we evaluated the role of gender in hematology clerkship in Fujian Medical University. We used either LBL or MTBL teaching method. Accordingly, practice score and case writing score between genders were analyzed to evaluate the difference of gender.

\section{Methods}

2.1. Female and Male Foreign Medical Student. Fujian Institute of Hematology (Fujian Provincial Key Laboratory on Hematology) and Department of Hematology Fujian Medical University Union Hospital organized this study on clerkship education between genders. Generally, this is a sixyear Fujian Medical University undergraduate program for foreign students majoring in clinical medicine. According to this program, the fourth year is dedicated to hematology clerkships for two weeks (they study other topics in other departments, on average, every department for 2-3 weeks). Iron deficiency anemia, aplastic anemia, megaloblastic anemia, hemolytic anemia, acute and chronic leukemia, lymphoma, multiple myeloma, bleeding diseases, and idiopathic thrombocytopenic purpura were included within the scope of this study.

In this study, twenty-two foreign medical students were included, who were randomly assigned into MTBL or LBL group by a random number table. It is comparable for female and male students in those groups. Seminars and lectures were given in both MTBL and LBL. For the MTBL group, fourteen foreign medical students were assigned. Each MTBL team included two foreign African medical students and one Chinese postgraduate (the role of the Chinese participant was to facilitate language understanding). Eight foreign African medical students were assigned to the LBL method group, which also had a Chinese translator in the clinic but no actively involved in the teaching and learning.

2.2. Evaluation and Scores. Before clerkship, there was a written test organized by Fujian Medical University for theoretical knowledge. The scores were given to the teachers before LBL or MTBL. The scores after clerkship were described in results section. Generally, those scores include fifteen percent of Medical Record Writing, fifteen percent of Medical Record Analysis, fifteen percent of Practice Physical Examination, fifteen percent of Practice Basic Clinical Performance, twenty percent of Medical Ethics, and twenty percent of Discipline.

2.3. Lecture-Based Learning Method. Hematology courses of physical examination, leukemia, lymphoma, multiple myeloma, anemia, and so on were given. The students were taught in a demonstration room. Before the demonstration class, the patients were temporarily moved into the room. Firstly, the teacher demonstrated how to inquire medical history and perform physical examination and then provided the examination results to the students as a reference. Secondly, after the demonstration, a lecture explaining the clinical characteristics of the cases was given, as well as the special examination methods, the key to disease identification, diagnosis and treatment, the fundamental concepts for the diseases, and so on. Finally, after two weeks of clerkship, the students were tested, and the scores after clerkship were sent to the database in Fujian Medical University.

2.4. Mixed Team-Based Learning Method. Students were divided into 7 small teams. In each team, there were two foreign African medical students and one Chinese postgraduate. Similar to LBL, MTBL was conducted in the lectures as described above. It is different in the MTBL group that Chinese postgraduates intervened in all the process of clerkship education, including the preparatory phase, clerkship phase, and after-lecture/seminars phase. Generally, in the preparatory phase, the foreign students familiarized the course objectives, requirements, and the case information with Chinese postgraduates. In the clerkship phase, the Chinese postgraduates involved in the communication between patients and foreign students. Also, intrateam discussions were performed. A simulated closed-book test that contains 10 multiple-choice questions was given by Chinese postgraduates. The questions were on the disease identification, differential diagnosis, and medical treatment. After the lecture, the foreign students were encouraged to perform 
Physical Examination and Basic Clinical Practice with Chinese postgraduates.

2.5. Statistical Analysis. All the data were evaluated with GraphPad Prism version 7.0 (GraphPad Software, Inc.). Means and SE were compared by unpaired two-tailed Student's $t$-test.

\section{Results}

To stimulate students' interest in learning and improve their self-learning ability, hematology clerkship was required. There are cultural differences between the African and Chinese students. Thus, the difficulties experienced by foreign students were evaluated before the clerkship. We found that most of them were not satisfied with the large amount of curriculum materials provided. Also they were unable to concentrate during study time due to culture gaps. Some felt a lack of emotional support from Chinese teachers and medical school. Some had difficulties in communication with patients as well as understanding Chinese traditions and values. Given these, as our published previous works mention, we developed MTBL teaching methods where Chinese postgraduates intervened in the education process, such as the preparatory phase, clerkship phase, and after-lecture phase in clinical clerkship. We found that this mixed team-based learning method overcomes some communication problems and has mutual benefit for foreigners and Chinese postgraduates.

In the preparatory phase, the Chinese postgraduates helped familiarize the foreign students with the course/lecture given by previous professors and the case information given by the teacher prior to the class. The Chinese postgraduates spend equal time and energy on male students and female students. During clerkship, the Chinese postgraduates helped the foreign students with the communication between patients and doctors. Also, intrateam discussions were performed. A simulated closed-book test containing 10 multiplechoice questions which focused on disease identification, diagnosis, and treatment choice was given by the Chinese postgraduates. After the lecture, the students were encouraged to contact the Chinese postgraduates if any clinical problem they encounter in clinical practice.

Next, we evaluated the role of gender in hematology clerkship. We found that the test scores before clerkship in males and females were not significantly different (Table 1), and after the hematology clerkship, the male group had no difference with female group in practice, case writing, and total score (Figures $1(\mathrm{a})-1(\mathrm{c}), P>0.05)$. Subgroup analysis in the LBL and MTBL groups showed that the scores in practice test scores, case-writing scores, and total scores were not significantly different between male and female students (Figures 2 and 3, $P>0.05$ ). These results indicate that there are no gender differences in foreign medical students' hematology clerkship.

\section{Discussion}

Gender differences in academic performance have become difficult to overlook. Most studies show that, generally, females
TABLE 1: Scores of the students before clerkship.

\begin{tabular}{lcc}
\hline Group & Number & Test scores before clerkship \\
\hline Male & 8 & $76.62 \pm 8.26$ \\
Female & 14 & $73.92 \pm 4.45$ \\
\hline
\end{tabular}

do better in medical school than males. To be specific, females achieved higher grades and completed high school at a higher rate as compared to males [10-12]. However, existing evidence of differences by gender in clerkship is weak. There is no theory, in hematology clerkship or otherwise, that empiricists can cite to explain the potential gender differences. Many Chinese medicine medical schools offer courses on international health and enroll international students [13]. Fujian Medical University began to recruit clinical medical undergraduate students in 1998. Majority of the foreign undergraduates come from Africa. The curriculum and teaching plan for foreign students were in accordance with the domestic medical undergraduates. Also, given the difference between foreigner and Chinese, there was some small difference, such as the hematology clerkship for foreigners was two weeks instead of one week for Chinese undergraduates. The Fujian Institute of Hematology has undertaken the teaching and clerkship of hematology for foreign medical students since 1998. In clinical practice, clerkship meets communication issues. Hematology studies about blood, the blood-forming organs, and blood diseases. Hematologists need to be at labs viewing blood films and bone marrow slides and interpreting results. Learning of hematology includes both clinical case and laboratory experience, which increases the difficulty of communication. Although, the hematological clerkship in Fujian Institute of Hematology mainly focuses on clinical cases, it still requires that international students not only have basic knowledge of hematology, but also good communication skills. It is commonly believed that females are better at communication; however, our study showed no gender differences in clinical practice.

The mission of education is to help students develop knowledge and skills appropriate to and necessary for their future careers. In our study, the enrolled foreign African medical students have very strong willingness to learn. Similar to team-based learning [14], MTBL is beneficial for teamwork and collaboration between students and showed greater effect than LBL in practice [5]. Some people believe that female students outperform males in most academic activities during the course of their education. However, in our study, no differences between genders have been found.

The lack of difference between genders as shown in our results may be surprising. One may argue that there might be gender difference between different teaching methods. In other words, it is still unknown whether there were differences between the 2 methodology cohorts; thus, we performed subgroup analysis. In the subgroup analysis, the similarity in genders appeared again. We believe that clerkship organized in group form, both in LBL and MTBL, 


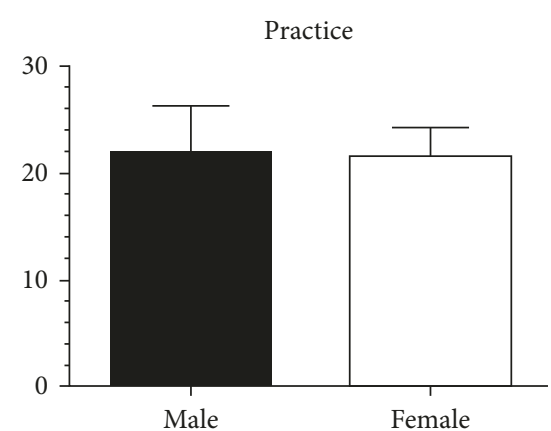

(a)

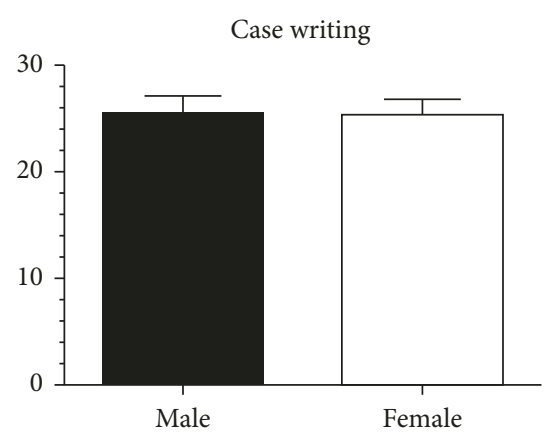

(b)

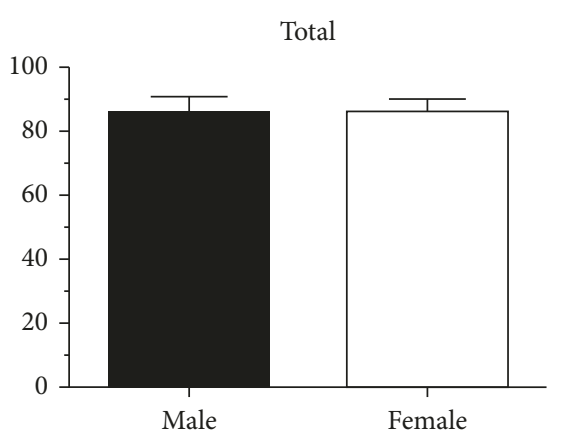

(c)

Figure 1: Comparison of scores at the end of the clerkship. The test scores between genders are represented. (a) Practice scores. (b) Casewriting scores. (c) Total scores.

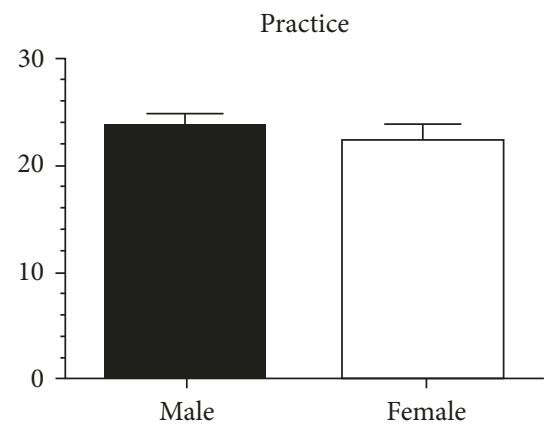

(a)

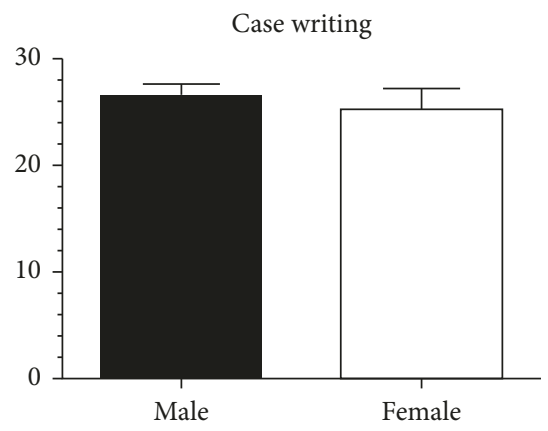

(b)

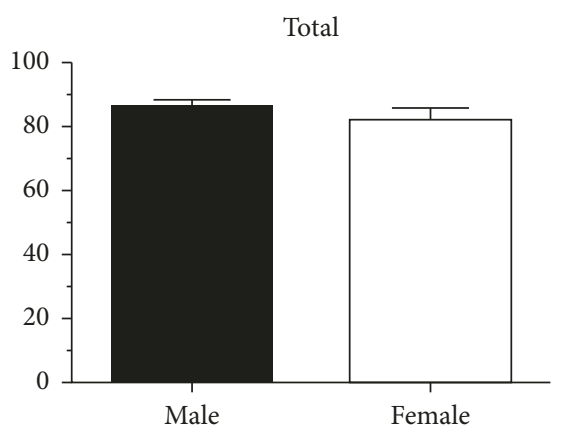

(c)

Figure 2: Comparison of scores in LBL at the end of the clerkship. The test scores between genders are represented. (a) Practice scores. (b) Case-writing scores. (c) Total scores.

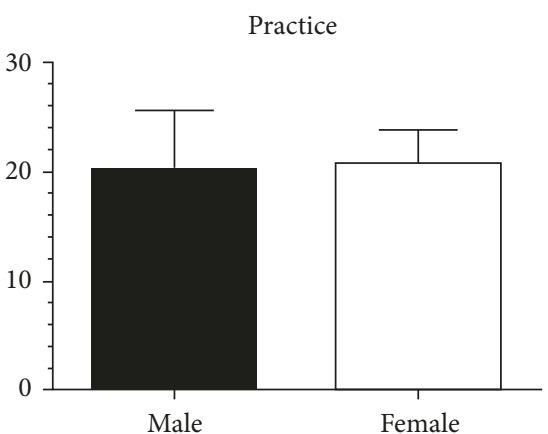

(a)

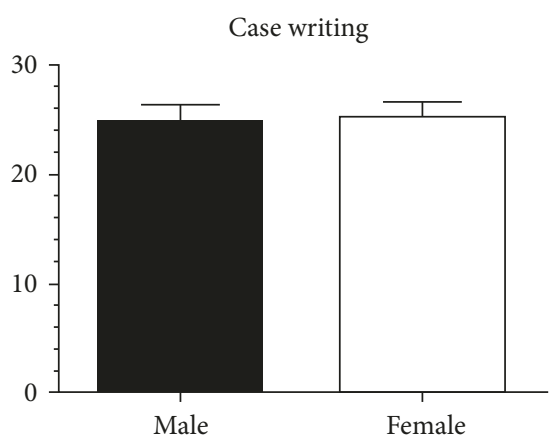

(b)

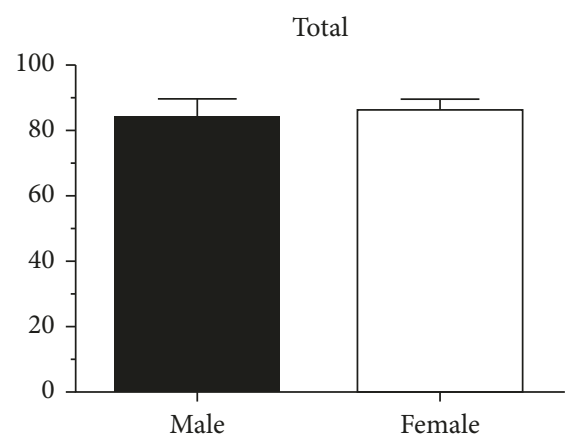

(c)

Figure 3: Comparison of scores in MTBL at the end of the clerkship. The test scores between genders are represented. (a) Practice scores. (b) Case-writing scores. (c) Total scores.

enhanced interactions between genders and compensated for the differences in communication. And there is no difference in gender between LBL and MTBL.

Finally, our results demonstrated similar scores in hematology clerkship between male and female students, which were not related to teaching methods. Our study only enrolled twenty-two students, and the study was short ( 2 weeks) as well. For foreign African medical students, the hematology clerkship is a small part of internal medicine and is only a two-week session. Whether gender plays a role in other subjects, such as neurology, gastroenterology, and so on, needs to be addressed with further studies.

\section{Conclusion}

There are no performance differences between foreign male and female students in hematology clerkship. 


\section{Conflicts of Interest}

The authors declare that there are no conflicts of interest regarding the publication of this paper.

\section{Authors' Contributions}

Qing Li and Jianhui Chen contributed equally to this article.

\section{Acknowledgments}

This work was supported by the Youth Project of National Natural Science Foundation (Grant no. 81200400), National Social Science Foundation of China (Grant no. 14CFX031), Top-Notch Innovative Talents Project of Fujian (Grant nos. 2016Y9025, 2016J06018, and 2012J05148), Fujian Medical University Teaching Reform Project (Y17005), and Fujian Provincial Health and Family Planning Commission Youth Research Project (2017-1-6).

\section{References}

[1] F. Paidar, A. Amirhooshangi, and R. Taghavi, "Gender differences in students' mathematics self-concept and academic burnout," International Journal of School Health, vol. 4, no. 1, p. e39351, 2016.

[2] A. Bleakley, "Gender matters in medical education," Medical Education, vol. 47, no. 1, pp. 59-70, 2013.

[3] M. Alers, L. van Leerdam, P. Dielissen, and A. Lagro-Janssen, "Gendered specialities during medical education: a literature review," Perspectives on Medical Education, vol. 3, no. 3, pp. 163-178, 2014.

[4] M. Mansh, W. White, L. Gee-Tong et al., "Sexual and gender minority identity disclosure during undergraduate medical education: "in the closet" in medical school," Academic Medicine, vol. 90, no. 5, pp. 634-644, 2015.

[5] K. Arnaoutakis, M. Anders, and K. Berry, "Simulating four essential conversations with hematology/oncology trainees: a qualitative evaluation," Journal of Cancer Education, vol. 31, no. 1, pp. 55-62, 2015.

[6] J. Liu, Y. X. Chen, X. R. Ma, H. E. Ai-Li, and X. M. Cao, "Preliminary application and problem analysis of bilingual teaching model in clinical practice of hemotology," Researches in Medical Education, vol. 9, no. 10, pp. 1358-1360, 2010.

[7] G. Norman, "Research in clinical reasoning: past history and current trends," Medical Education, vol. 39, no. 4, pp. 418-427, 2005.

[8] C. Enarson and L. Cariaga-Lo, "Influence of curriculum type on student performance in the United States Medical Licensing Examination Step 1 and Step 2 exams: problem-based learning vs. lecture-based curriculum," Medical Education, vol. 35, no. 11, pp. 1050-1055, 2001.

[9] X. Chen, B. Chen, X. Li, Q. Song, and Y. Chen, "Mutual benefit for foreign medical students and Chinese postgraduates: a mixed team-based learning method overcomes communication problems in hematology clerkship," Biochemistry and Molecular Biology Education, vol. 45, no. 2, pp. 93-96, 2016.

[10] M. J. Zembar and L. B. Blume, "Gender and academic achievement," in Middle Childhood Development: A Contextual Approach, pp. 212-215, Prentice Hall, Upper Saddle River, NJ, USA, 2nd edition, 2009.

[11] D. Booth, S. Elliott-Johns, and F. Bruce, Boys' Literacy Attainment: Research and Related Practice, Centre for Literacy at Nipissing University, Ontario, Canada, 2012.
[12] N. M. Fortin, P. Oreopoulos, and S. Phipps, Leaving Boys Behind: Gender Disparities in High Academic Achievement, University of British Columbia, University of Toronto, Dalhousie University, and CIFAR, 2012.

[13] D. Yang, On the Eight Changes of Higher Education Institutions Reform in China, Peking University, Beijing, China, 2008.

[14] B. M. Thompson, V. F. Schneider, H. Paul et al., “Team- based learning at ten medical schools: two years later," Medical Education, vol. 41, no. 3, pp. 250-257, 2007. 


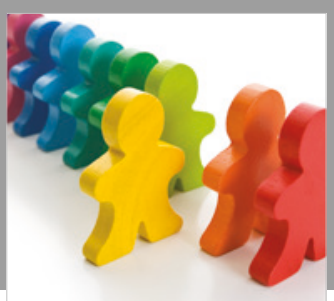

Autism

Research and Treatment
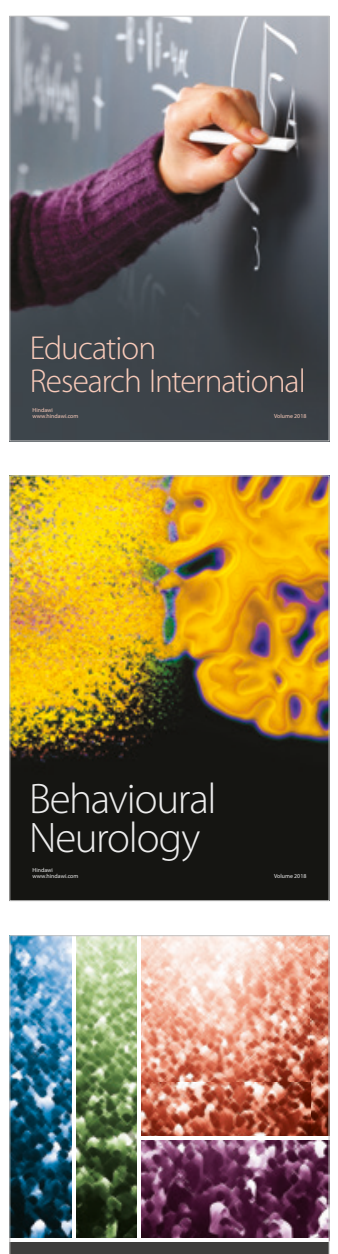

International Journal of

Population Research

$\underline{-m}$

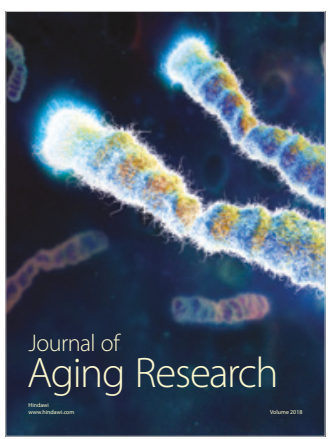

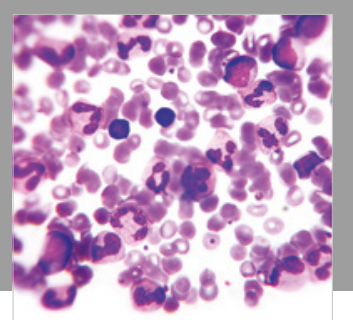

Pathology

Research International$$
=
$$

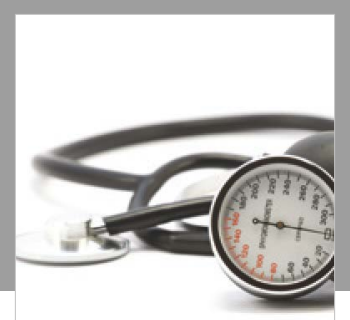

Nursing

Research and Practice

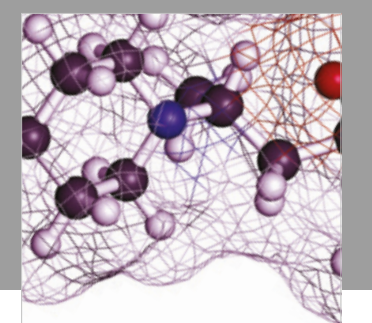

Pain

Research and Management

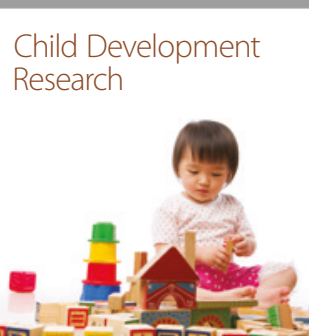

बाD

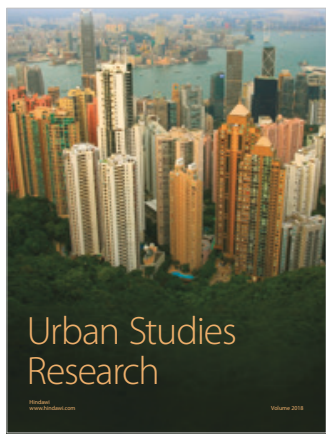

\section{Hindawi}

Submit your manuscripts at

www.hindawi.com
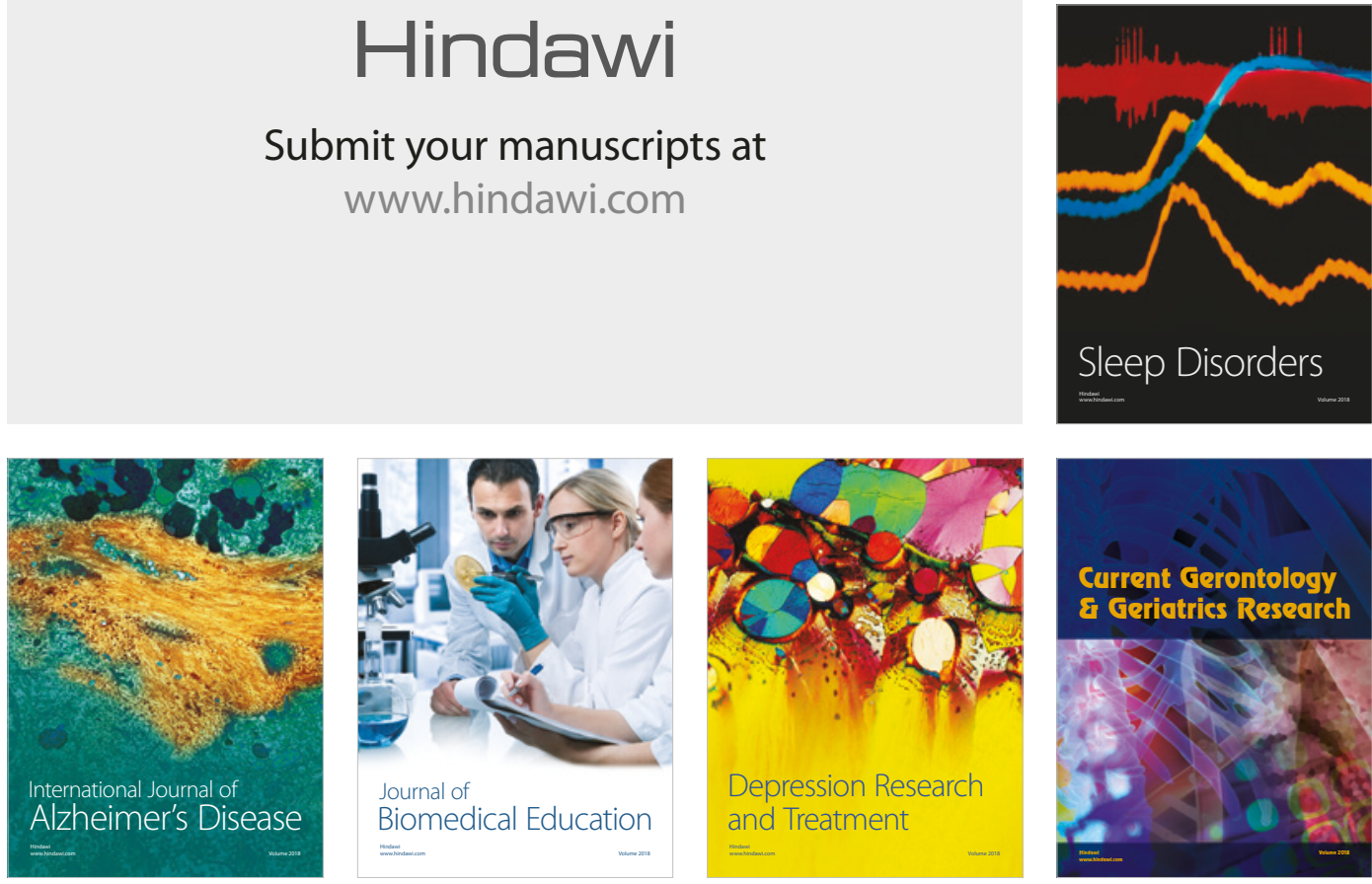

Journal of

Biomedical Education

$=$

smman

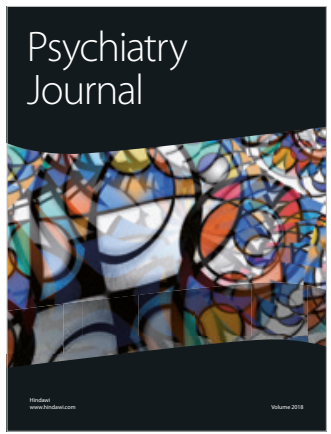

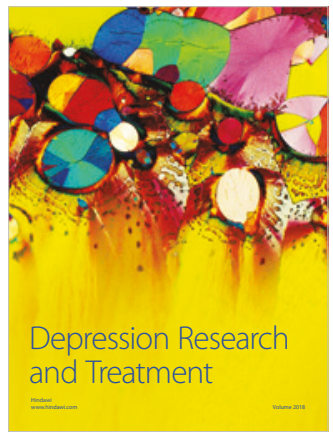
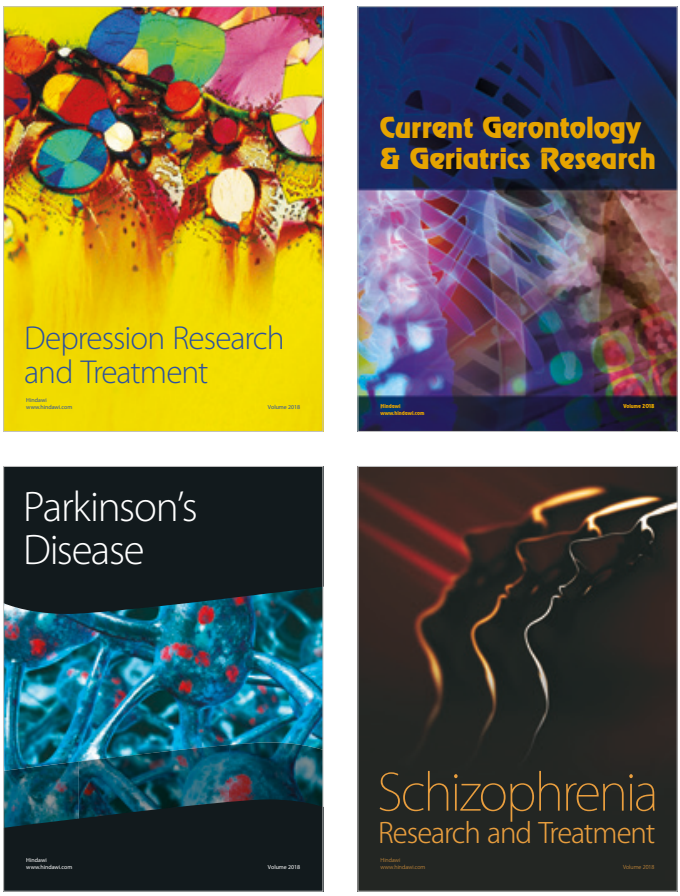